Check for updates

Cite this: J. Mater. Chem. C, 2019, 7, 170

Received 15th September 2018, Accepted 9th November 2018

DOI: $10.1039 / \mathrm{c} 8 \mathrm{tc} 04677 \mathrm{~h}$

rsc.li/materials-c

\section{All-optical switching in dye-doped DNA nanofibers $\dagger$}

\author{
Adam Szukalski, (D) ab Maria Moffa, ${ }^{\mathrm{b}}$ Andrea Camposeo, (D) ${ }^{\mathrm{b}}$ Dario Pisignano (D) $* \mathrm{bc}$ \\ and Jaroslaw Mysliwiec (D)
}

\begin{abstract}
All-optical switches are introduced which are based on deoxyribonucleic acid (DNA) in the form of electrospun fibers, where DNA is semi-intercalated with a push-pull, luminescent nonlinear pyrazoline derivative. Optical birefringence is found in the organic nanofibers, with fully reversible switching controlled through continuous-wave laser irradiation. The photoinduced signal is remarkably large, with birefringence highlighted by optically-driven refractive index anisotropy approaching 0.001 . Sub-millisecond characteristic switching times are found. Integrating dye-intercalated DNA complex systems in organic nanofibers, as a convenient and efficient approach to template molecular organization and control it by external stimuli, might open new routes for realizing optical logic gates, reconfigurable photonic networks and sensors through physically-transient biopolymer components.
\end{abstract}

\section{Introduction}

The nonlinear optical properties of bulk organic materials and micro-architectures based on them are generally associated with their response far from resonance, including refractive index changes, which originates mostly from the electronic cloud deformation occurring at the molecular scale. Indeed, the potentially large, fast nonlinear behaviour of various organic systems is related with $\pi$-electron system delocalization ${ }^{1}$ and is non-resonant in its nature. However, a large enhancement of the nonlinear behaviour might occur when excitation light is close to resonance, with response timescales up to $10^{-2} \mathrm{~s}$. Importantly, additional control of the nonlinear optical properties of organic systems is provided by the materials embedding the photoactive molecules. Examples of matrix compounds which can host organic molecules relevant in this framework include biopolymers like deoxyribonucleic acid (DNA), ${ }^{2-4}$ possibly

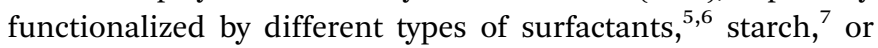
collagen. ${ }^{8}$

In particular, DNA which has been studied almost exclusively by biologists over the past half-century, ${ }^{9}$ is now a well-established polymer for developing a variety of processes and devices in photonics and nanotechnology. ${ }^{9,10}$ It was shown that DNA modified with surfactant molecules, which is insoluble in water

\footnotetext{
${ }^{a}$ Faculty of Chemistry, Wroclaw University of Science and Technology, Wybrzeze Wyspianskiego 27, 50-370 Wroclaw, Poland

${ }^{b}$ NEST, Istituto Nanoscienze-CNR, Piazza S. Silvestro 12, I-56127 Pisa, Italy ${ }^{c}$ Dipartimento di Fisica, Università di Pisa, Largo B. Pontecorvo 3, I-56127 Pisa, Italy. E-mail: dario.pisignano@unipi.it

$\dagger$ Electronic supplementary information (ESI) available. See DOI: 10.1039/c8tc04677h
}

but easily processable in organic solvents, leads to optical materials with significant transparency in the visible range. ${ }^{11,12}$ Also, doping such a matrix with light-emitting molecules changes its properties and might allow blends to be obtained which show amplified spontaneous emission, ${ }^{13}$ lasing ${ }^{14}$ or random lasing. ${ }^{15}$ Moreover, doping with photochromic molecules makes the resulting composite suitable for fast holographic recording due to the dye semi-intercalation. ${ }^{12}$ Modified DNA complexes are being also used in organic electronics to realize transistors, or light-emitting diodes. ${ }^{16-25}$ The by far largest amount of studies applying these systems in photonics and electronics are based on film structures. However, the random and variable distribution of dye molecules, which is generally achieved in films, might prevent the effective and coherent addressing of individual molecules or ensembles made of them, which is highly important in order to exploit molecular functions in practically usable devices. In this respect, integrating dyeintercalated DNA complex systems in micro- and nanostructures could open new possibilities for studying their photo-physical properties, for managing them in a coherent way, and for templating their organization making the modification of molecular status by external stimuli much more convenient and efficient, also due to the greatly enhanced surface-to-volume ratio. Other important aspects are related to the low-cost and potential easy processability of DNA-based nanostructures, especially nanofibers. In these systems, thanks to the unique double helix structure and interaction between each host DNA chain and low-molar mass dyes (guests), fully reversible and stable all-optical bio-switches might be feasible. So far, various nonlinear mechanisms have been introduced for all-optical switching of light beams, such as saturable absorption and optical 
(a)

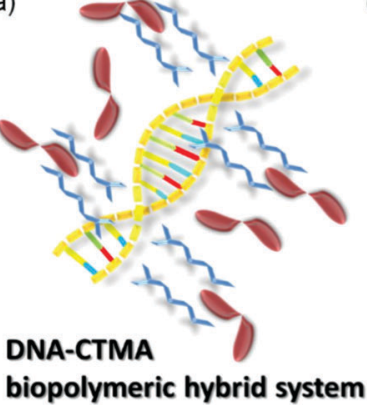

(b)

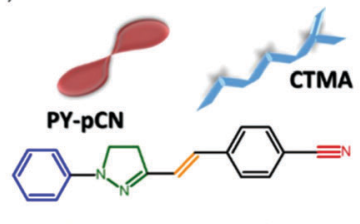

DONOR $\rightarrow$ PYRAZOUINE RING $\rightarrow$ $\rightarrow$ DOUBLE BOND $\rightarrow$

$\rightarrow$ ACCEPTOR

\section{biopolymeric hybrid system}

(c)



Fig. 1 (a) Scheme of the photoisomerizable dye semi-intercalation in the DNA-CTMA matrix. (b) Chemical structure of the photoactive medium (PY-pCN). Arrows in the schematics indicate intramolecular charge transfer paths for electrons. (c) Steps of PY-pCN synthesis. NBS: N-bromosuccinimide; AlBN: azobisisobutyronitrile; $\mathrm{PPh}_{3}$ : triphenylphosphane.

limiting, ${ }^{26-30}$ nonlinear refractive index and photoinduced birefringence, ${ }^{6,31,32}$ thermo-optic effects, ${ }^{33}$ and using organics or 2D nanomaterials either in liquids or in films and nanosheets. Nevertheless, switches based on organic nanofibers are still poorly studied, and optical properties, such as refractive index, have never been controlled by external light, which is critically important for all-optical interconnects.

Here, we demonstrate all-optical switching in organic fibers consisting of DNA functionalized with cetyltrimethylammonium chloride surfactant (DNA-CTMA) and doped with a nonlinear pyrazoline derivative chromophore (PY- $p \mathrm{CN})$ as a typical push-pull molecular system (Fig. 1a). Fibers and excitation laser light serve as templates for molecular ordering leading to optical anisotropy and driving the system into two well-defined states used for the switch construction. Based on the achieved light-controlled birefringence, a great potential is suggested for these materials for fast all-optical switching applications. These might include remotelyreconfigurable photonic networks and sensors, ${ }^{34}$ opto-logic gates and multiplexers, ${ }^{35,36}$ and physically-transient optoelectronic components ${ }^{2}$ based on natural biopolymers.

\section{Results and discussion}

Previous studies found that the specific interaction between the DNA and photochromic azo-molecules (semi-intercalation) leads to a fully-reversible photoisomerization which is much faster than that of typical host-guest systems. ${ }^{6,37-42}$ Thanks to this unique structure promoted by the bio-organic matrix (Fig. 1b), it is possible to obtain semi-hooking of the dye molecules and to straightforwardly control their spatial arrangement by polarized light irradiation, thus causing dynamic and fullyreversible modulation of refractive index. The so-achieved, photo-controlled system features two well-defined and reversible states. PY- $p$ CN, which is a push-pull, $\pi$-conjugated compound featuring intramolecular charge transfer, was achieved by the synthetic route shown in Fig. 1c and detailed in the ESI $\dagger$ (molecular characterization is reported in Fig. S1 and S2). ${ }^{42}$ Recently, pyrazoline derivatives were utilized by us for light amplification (in laser action or random lasing), ${ }^{3,43-45}$ multiphoton absorption, ${ }^{42,46}$ and generation of light at higher harmonics, ${ }^{47,48}$ and their structure was found to be modified by light in a reversible way through trans or cis states. ${ }^{49,50}$

Electrospun PY- $p$ CN/DNA-CTMA mats consist of mainly ribbon-shaped microfibers (Fig. 2a) with roughly bi-modal distributions in terms of transversal size peaked at about $250 \mathrm{~nm}$ and at about $1.2 \mu \mathrm{m}$, respectively (Fig. S3, ESI $\dagger$ ). The absorption spectrum of the active material, with the laser lines used for pump and probe experiments, is presented in Fig. S4 in the ESI. $\dagger$ In our fibers, the overall degree of dopant alignment can be easily controlled by linearly polarized laser light, thus leading to refractive index anisotropy. Indeed, the fiber-embedded photoswitchable molecules can be oriented independently on the filament direction, the transition from the initially-isotropic system to an anisotropic one being promoted by laser excitation.

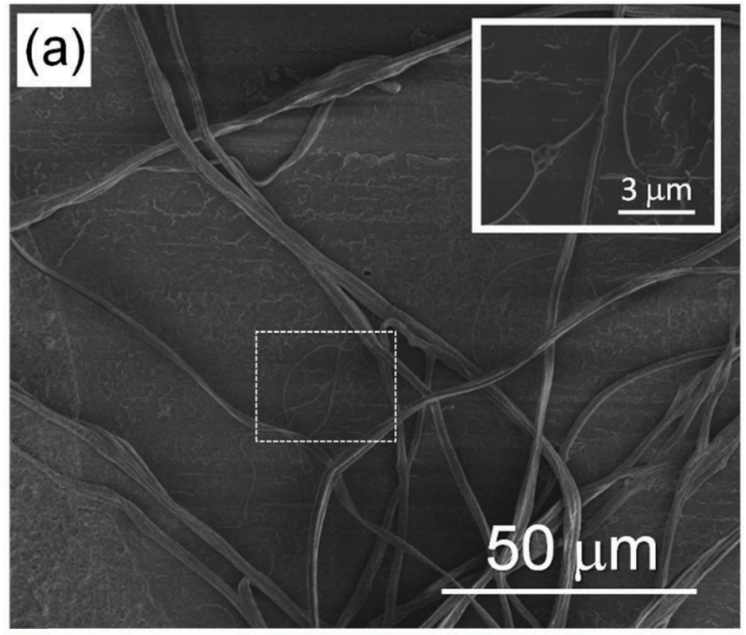

(b)

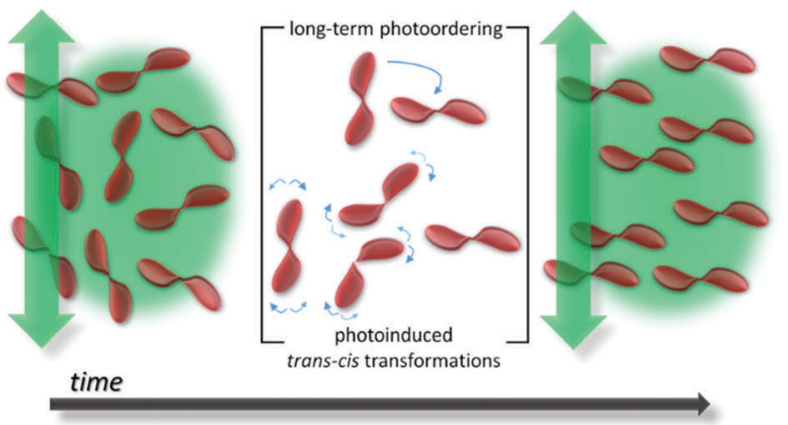

Fig. 2 (a) SEM micrograph of PY-pCN-doped DNA-CTMA fibers. Inset: Higher magnification view of the region highlighted by the dashed rectangle, showing fibers with diameters down to $>100 \mathrm{~nm}$. (b) Scheme of the photoinduced optical birefringence generated through molecular alignment in electrospun nanofibers, induced by linearly polarized laser light. Vertical green arrows: direction of light polarization; single blue arrow: long-term photo-ordering; doubled blue arrows: photo-induced trans-cis-trans transitions. 
Two regimes can be distinguished in this respect, namely shortand long-term photo-ordering, as schematized in Fig. $2 \mathrm{~b}$.

In the short-term $\left(10^{-9}\right.$ to $\left.10^{-3} \mathrm{~s}\right),{ }^{51}$ proper photoinduced molecular transitions like trans-cis-trans photoisomerizations should be considered. For this process to take place, the excitation wavelength should be within the absorption range of the active medium, and there should be enough free volume in the matrix to enable the involved transition. In parallel, thermal relaxation occurs converting the metastable cis-form to the stable trans-isomer, and continuously leading the system towards a more isotropic arrangement (i.e. to a lowest-energy thermodynamic state) over timescales typically ranging from $10^{-3} \mathrm{~s}$ to a few minutes. ${ }^{51-53}$ The onset of significant photoalignment at this timescale can be highlighted modulating the pump beam at frequencies above a few hundreds of $\mathrm{Hz}$, and measuring the refractive index anisotropy by using an oscilloscope in AC mode in pump-probe experiments (Fig. S5, ESI $\dagger$ ). Following a very large-number of the so-defined photoinduced trans-cis-trans transitions, a photostationary state is achieved and long-term photo-ordering is established (highlighted by a DC mode in the detecting oscilloscope), with in principle all the dopants oriented perpendicularly with respect to the laser light polarization direction and timescales from seconds to days. This is promoted by rotational diffusion ${ }^{54}$ of the nonlinear chromophores as a consequence of the coupling with their local microenvironment (generally constituted by the solvent for solutions, and by the polymer matrix for solidstate samples). The photoinduced birefringence measured in PY- $p$ CN/DNA-CTMA fibers and its dependence on the excitation intensity ( $I$ ) are shown in Fig. 3 for long-term photo-ordering. A clear linear behaviour is found for the photoinduced refractive index anisotropy $(\Delta n)$, i.e. $\Delta n(I)=n_{2} I$ with $n_{2}=2.0 \times 10^{-10} \mathrm{~m}^{2} \mathrm{~W}^{-1}$, without evidence of signal saturation in the investigated range of pump intensities. This indicates remarkable photostability of the realized optical material. By increasing the intensity of


anisotropy, $\Delta n$, approaches values of the order of 0.001 (Fig. 3b).

Similar to the optical Kerr effect, the intensity-induced linear birefringence can be described by an effective thirdorder nonlinear optical susceptibility, ${ }^{55-57} \chi_{\mathrm{eff}}^{(3)} \cong n_{2} n_{0}{ }^{2} \varepsilon_{0} c$, where $n_{0}$ denotes the refractive index of the system without excitation from the pump beam $\left(n_{0}=1.5\right),{ }^{58} \varepsilon_{0}$ is the dielectric constant and $c$ the light velocity, both in vacuum conditions. In electrospun PY- $p$ CN/DNA-CTMA fibers, the third-order nonlinear optical susceptibility was estimated to be of the order of $1 \times 10^{-12} \mathrm{~m}^{2} \mathrm{~V}^{-2}$, in line with those found in well-established organic systems embedding nonlinear chromophores. ${ }^{49,50}$

As unprecedented evidence of such all-optical switching effect in electrospun organic fibers, these findings make such systems a promising alternative against spiropyran/merocyanine, ${ }^{59}$ film-embedded azobenzene chromophores, ${ }^{52,60}$ polymethine compounds, ${ }^{61,62}$ and conjugated polycyanine. ${ }^{63}$ Exemplary, monoexponential increase and decay behaviours for the long-term photo-ordering in the fibers are shown in Fig. S6 (ESI $\dagger$ ), indicating symmetric response during switching 'on' and 'off' the system, with characteristic time for achieving the stationary state of (a)

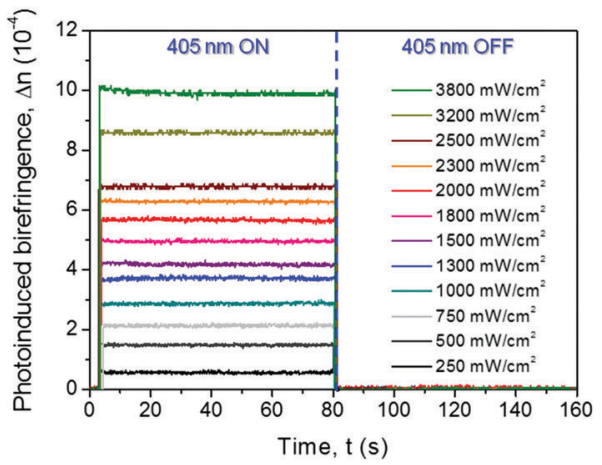

(b)

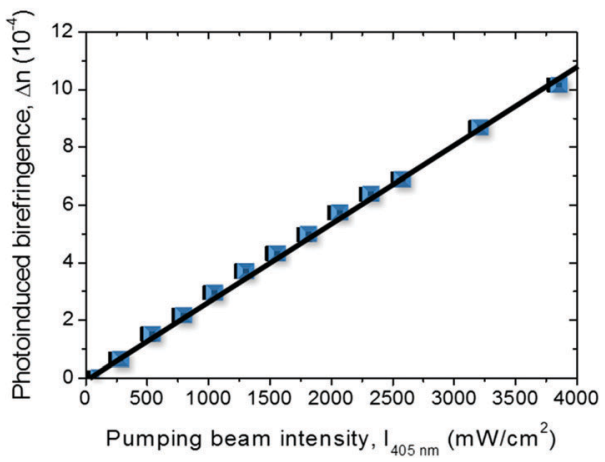

Fig. 3 Dependence of measured photoinduced birefringence on power excitation intensity from the pump beam (long-term photo-ordering). (a) Switching behaviour. (b) Dependence of the photoinduced refractive index anisotropy (birefringence), $\Delta n$, on the pumping beam intensity.

photo-ordering of about $0.4 \mathrm{~ms}$. In Fig. 4a we present the photoinduced birefringence acquired with the oscilloscope in DC mode and a pump beam modulator. The remarkable signal stability at long times is highlighted in Fig. 4b. Even upon multiple photoinduced birefringence processes in the PY- $p \mathrm{CN} /$ DNA-CTMA fibers, $\Delta n$ can be re-built quickly and, what is crucial for realizing optical switching devices, without appreciable efficiency losses.

To rationalize the molecular switching process more in depth and investigate the range of switching frequency accessible through electrospun PY-pCN/DNA-CTMA nanofibers, we investigated the correlation between photoinduced birefringence and laser light modulation frequency (Fig. 5a). When the modulation frequency is roughly $300 \mathrm{~Hz}$ or lower, laser irradiation intervals are long enough to approach the photostationary state and a longterm optical anisotropy generation. This is evidenced by the stable values of the photoinduced birefringence measured for pump modulations in the range of frequency $50-300 \mathrm{~Hz}$ (Fig. 5a). For faster modulations, the maximum photoinduced birefringence decreases, a result suggesting a predominant role of trans-cistrans photoisomerization and thermal relaxation in long-term photo-ordering. For modulation at $800 \mathrm{~Hz}$, a $\Delta n$ of 0.00017 is found, highlighting a switching behaviour still significant upon approaching $\mathrm{kHz}$ frequencies (Fig. 5b). The fast-responding system shows increase and decay times $\tau_{\mathrm{AC}}(\mathrm{inc})=\tau_{\mathrm{AC}}(\mathrm{dec})=0.35 \mathrm{~ms}$ at a modulation frequency of $200 \mathrm{~Hz}$ (Fig. 5c and d). Such characteristic switching times are an order of magnitude lower than the ones observed for the PY- $p \mathrm{NO}_{2}$ embedded in PMMA. ${ }^{49}$ 
(a)

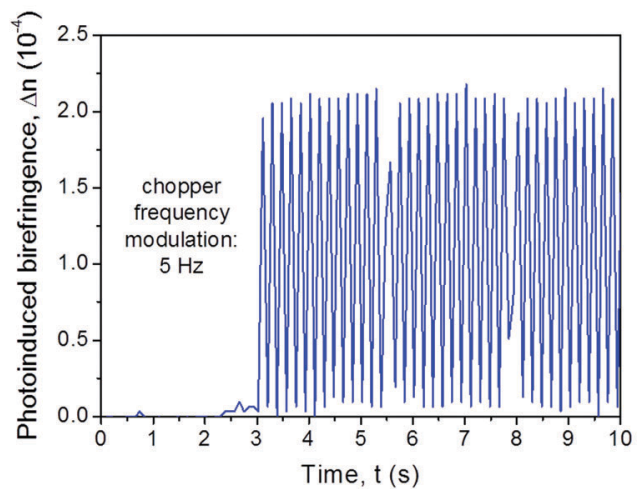

(b)

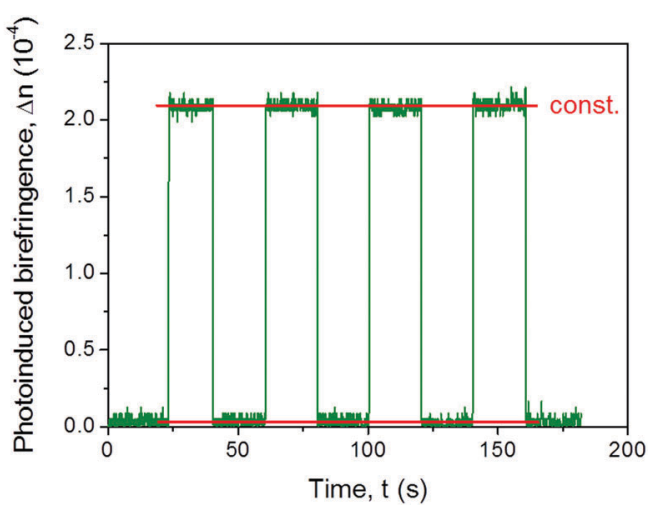

Fig. 4 (a) $\Delta n$ values measured upon applied light modulation and (b) the multiple photoinduced birefringence process in PY-pCN/DNA-CTMA fibers $\left(I_{\text {pump }}=750 \mathrm{~mW} \mathrm{~cm}^{-2}\right.$ ).

Hence, the nanostructured biopolymer matrix here developed allows sub-millisecond all-optical switching to be achieved. This is relevant for various spectroscopic, imaging and manufacturing applications, where either intensity or phase modulation of light beams is required. ${ }^{60,64}$

The realization of DNA fibers by electrified jets, ${ }^{65}$ promoting orientation of macromolecules within individual bio-polymer filaments, might be especially relevant in stabilizing photoinduced optical anisotropy. Indeed, the higher molecular order induced by jet elongation during electrospinning, leading to fibers with polymer chains prevalently oriented along the longitudinal axis of the filaments, directly favour the increase of optical anisotropies, ${ }^{66-68}$ a property typically enhanced in thinner electrospun fibers. ${ }^{68}$ The complex interplay of these mechanisms with the photo-alignment of the initially-isotropic embedded chromophores might make dye-doped DNA fibers efficient, repeatable and fast-responsive optical switches, with remarkable non-linear optical signals and stability. Moreover, the possibility of controlling the molecular order in electrospun nanofibers by processing parameters and ultimately through the fiber transversal size ${ }^{68}$ could constitute an additional variable for tailoring the nonlinear optical properties of embedded compounds. This approach can be pretty useful for photonic computing architectures, where logic gates are required to control data transfer. For these applications, functionalized DNA in anisotropic template nanostructures appears unique in its association with semi-intercalation of nonlinear chromophores, and highly effective for supporting molecular photoalignment.

\section{Materials and methods}

\section{Materials}

We used DNA functionalized with a CTMA surfactant (SigmaAldrich) and doped with the luminescent and photoisomerizable chromophore (E)-2-(2-(1-phenyl-4,5-dihydro-1H-pyrazol-3-yl)vinyl)benzonitrile (PY- $p \mathrm{CN}$ ) (Fig. S2, ESI $\dagger$ ). For realizing PY-pCN-doped DNA-CTMA fibers, the DNA-CTMA complex was dissolved in butanol with a $4 \%$ dry mass weight ratio $(\mathrm{w} / \mathrm{w})$ and stirred for $24 \mathrm{~h}$. A second solution was obtained using the same solvent and PY- $p$ CN at $2 \%$ (also $w / w$ ) dye concentration. A mixture was then obtained by suitable quantities of the two solutions to reach a $1 \%$ concentration of nonlinear chromophore in the dry mass of the biopolymer, which was electrospun using a feeding rate of $0.5 \mathrm{~mL} \mathrm{~h}^{-1}$, a distance between the spinning tip and collector surface of $20 \mathrm{~cm}$, and an applied voltage of $10 \mathrm{kV}$. The morphology of the doped fibers was investigated by scanning electron microscopy (SEM, Nova NanoSEM 450, FEI) with an accelerating voltage of $3 \mathrm{kV}$ and an aperture size of $30 \mu \mathrm{m}$, following thermal deposition of $5 \mathrm{~nm}$ of $\mathrm{Cr}$ (PVD75, Kurt J. Lesker Co.). The average diameter of the fibers was calculated from the SEM micrographs using imaging software.

\section{Spectroscopy}

All-optical switching processes were investigated by a pumpprobe experimental set-up (Fig. S5 in the ESI $\dagger$ ), described in detail elsewhere. ${ }^{49,50}$ Briefly, we used two CW laser light sources with $405 \mathrm{~nm}$ and $633 \mathrm{~nm}$ wavelength to generate pump and probe beams, respectively. The two beams were converged at one point located on the sample. For characterizing chromophore photoalignment at long time-scales (associated with the quasi-static component of the photoinduced refractive index anisotropy, $\Delta n$ ), the pump beam was switched "on" and "off" manually to promote optical anisotropy (pump laser ON) or let the system go through thermal relaxation processes under darkness (pump laser OFF), respectively. A cross-polarized system was used, equipped with a sensitive photodiode combined with an oscilloscope in DC mode to acquire the above dynamics. For assessing the dynamic component of photoinduced birefringence, namely for characterization at a shorter timescale, a mechanical chopper with controlled frequency modulation was embedded in the experimental set-up to modulate the pump intensity, and the analysing oscilloscope set in AC mode. The photoinduced refractive index anisotropy $(\Delta n)$ is determined as a function of time $(t)$ and excitation intensity $(I)$ at the same time, and correlated with phase changes $(\Delta \varphi$, which is also function of $t$ and $I$ ), wavelength of the probe beam $(\lambda)$ and sample thickness $(d)$ as $^{49,50} \Delta n(I, t)=\lambda \Delta \varphi(I, t) / 2 \pi d$. The correlation between the intensities of incident $\left(I_{0}\right)$ and transmitted ( $I_{\text {trans }}$ ) probe light involved with the phase change and resulting refractive index anisotropy is given by: ${ }^{49,50} I_{\text {trans }}(t)=I_{0} \sin ^{2}[\pi d \Delta n(I, t) / \lambda]$. 
(a)

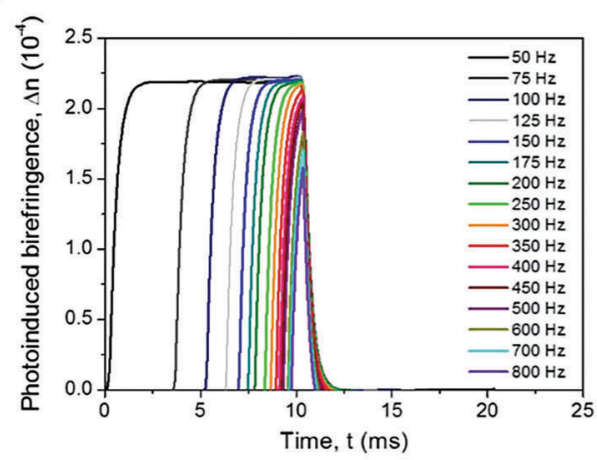

(c)

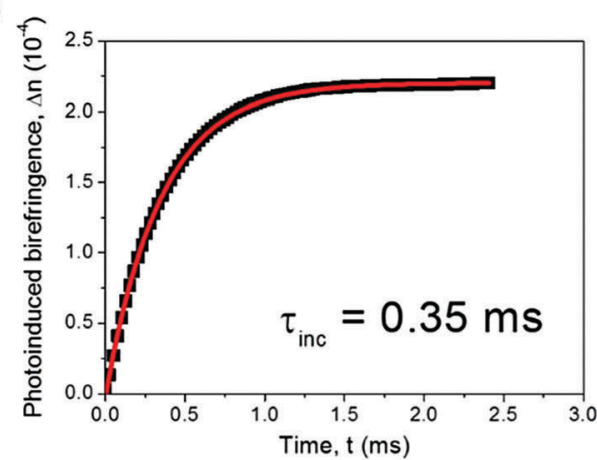

(b)

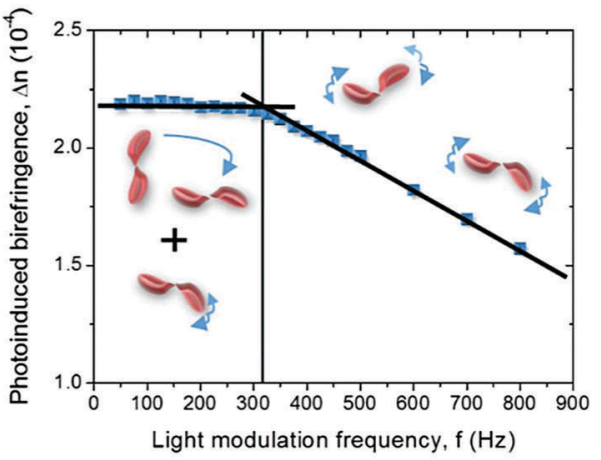

(d)

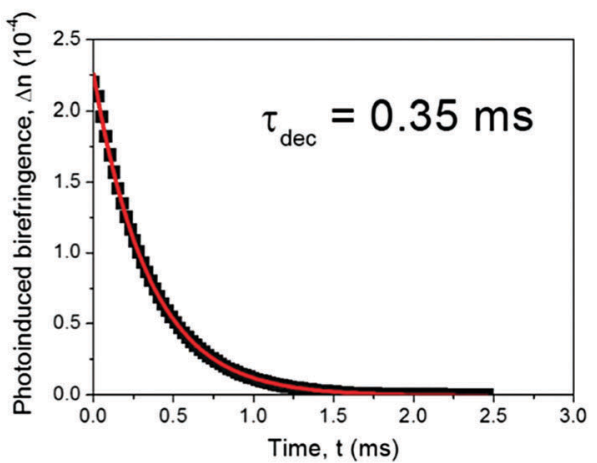

Fig. 5 Photodynamic changes of the induced birefringence in PY-pCN/DNA-CTMA fibers. (a) AC component of the photoinduced birefringence signal for various modulation frequencies of the pump light. (b) $\Delta n$ vs. modulation frequency. The vertical continuous line highlights the transition from longterm (modulation frequency $<300 \mathrm{~Hz}$ ) to short-term photo-ordering (modulation frequency $>300 \mathrm{~Hz}$ ). Lines superimposed to experimental data are guides for the eye. Corresponding birefringence increase (c) and decrease (d), with exponential fits of experimental results. Applied modulation frequency: $200 \mathrm{~Hz}$. $I_{\text {pump }}=750 \mathrm{~mW} \mathrm{~cm}^{-2}$.

This approach allows changes in the material at nanoscopic scales to be assessed by straightforward power measurement of the probe laser beam.

\section{Conclusions}

In summary, optical switches based on electrospun fibers made by properly-functionalized biological material have been proposed. DNA-CTMA doped with pyrazoline derivative features effective, stable, quick and fully-reversible modulations of the refractive index. Time constants associated with the involved dynamic changes (trans-cis-trans molecular transformations and long-term photoalignment) are $\tau_{\mathrm{AC}}($ inc $)=\tau_{\mathrm{AC}}(\mathrm{dec})=0.35 \mathrm{~ms}$, respectively, suggesting these fibrous materials as a valuable competitor of well-known azobenzene-based systems. The present optical switches based on a bio-matrix and pyrazoline derivative can be utilized for the design and development of efficient and fast sensing schemes, photonic logic operators, and organic multiplexers based on functional nanofibers.

\section{Conflicts of interest}

There are no conflicts to declare.

\section{Acknowledgements}

A. S. Supported by the Foundation for Polish Science (FNP). J. M. This work was financially supported by The National Science Centre, Poland (2016/21/B/ST8/00468) and by statutory founds of the Wroclaw University of Science and Technology. The research leading to these results has also received funding from the European Research Council under the European Union's Seventh Framework Programme (FP/2007-2013)/ERC Grant Agreement no. 306357 ("NANO-JETS") (D. P.) and under the European Union's Horizon 2020 Research and Innovation Programme (Grant Agreement no. 682157, "xPRINT") (A. C.). D. P. also acknowledges the support from the project PRA_2018_34 (“ANISE”) from the University of Pisa. Karolina Haupa is gratefully acknowledged for infrared spectroscopy on PY- $p$ CN.

\section{Notes and references}

1 D. S. Chemla and J. Zyss, Nonlinear Optical Properties of Organic Molecules and Crystals, Academic Press, New York, 1987.

2 A. Camposeo, P. Del Carro, L. Persano, K. Cyprych, A. Szukalski, L. Sznitko, J. Mysliwiec and D. Pisignano, ACS Nano, 2014, 8, 10893. 
3 I. Rau, A. Szukalski, L. Sznitko, A. Miniewicz, S. Bartkiewicz, F. Kajzar, B. Sahraoui and J. Mysliwiec, Appl. Phys. Lett., 2012, 101, 171113.

4 A. J. Steckl, Nat. Photonics, 2007, 1, 3.

5 E. M. Heckman, J. A. Hagen, P. P. Yaney, J. G. Grote and F. K. Hopkins, Appl. Phys. Lett., 2005, 87, 211115.

6 A. Miniewicz, A. Kochalska, J. Mysliwiec, A. Samoc, M. Samoc and J. G. Grote, Appl. Phys. Lett., 2007, 91, 041118.

7 K. Cyprych, L. Sznitko and J. Mysliwiec, Org. Electron., 2014, 15, 2218.

8 K. Cyprych, M. Janeczko, I. Rau, F. Kajzar and J. Mysliwiec, Org. Electron., 2016, 39, 100.

9 R. A. Goodnow, A Handbook for DNA-Encoded Chemistry: Theory and Applications for Exploring Chemical Space and Drug Discovery, Wiley, New Jersey, 2014.

10 J. G. Grote, D. E. Diggs, R. L. Nelson, J. S. Zetts, F. K. Hopkins, F. K. Ogata, J. A. Hagen, E. Heckman, P. P. Yaney, M. O. Stone and L. R. Dalton, Mol. Cryst. Liq. Cryst., 2005, 426, 3 .

11 E. M. Heckman, J. A. Hagen, P. P. Yaney, J. G. Grote and F. K. Hopkins, Appl. Phys. Lett., 2005, 87, 211115.

12 C. A. Lazar, F. Kajzar, I. Rau and A.-M. Manea, Synth. Met., 2016, 221, 120.

13 J. Mysliwiec, L. Sznitko, A. Miniewicz, F. Kajzar and B. Sahraoui, J. Phys. D: Appl. Phys., 2009, 42, 1.

14 Y. Kawabe and K.-I. Sakai, Nonlinear Opt., Quantum Opt., 2011, 43, 273.

15 L. Sznitko, A. Szukalski, K. Cyprych, P. Karpinski, A. Miniewicz and J. Mysliwiec, Chem. Phys. Lett., 2013, 576, 31.

16 Y.-W. Kwon, C. H. Lee, D.-H. Choi and J.-I. Jin, J. Mater. Chem., 2009, 19, 1353.

17 J. A. Hagen, W. Li, A. J. Steckl and J. G. Grote, Appl. Phys. Lett., 2006, 88, 171109.

18 D. Wanapun, V. J. Hall, N. J. Begue, J. G. Grote and G. J. Simpson, ChemPhysChem, 2009, 10, 2674.

19 E. M. Heckman, J. G. Grote, F. K. Hopkins and P. P. Yaney, Appl. Phys. Lett., 2006, 89, 181116.

20 J. Zhou, Z. Y. Wang, X. Yang, C.-Y. Wong and E. Y. B. Pun, Opt. Lett., 2010, 35, 1512.

21 Z. Yu, W. Li, J. A. Hagen, Y. Zhou, D. Klotzkin, J. G. Grote and A. J. Steckl, Appl. Opt., 2007, 46, 1507.

22 M. Leonetti, R. Sapienza, M. Ibisate, C. Conti and C. López, Opt. Lett., 2009, 34, 3764.

23 T. Sasaki, H. Ono and N. Kawatsuki, Jpn. J. Appl. Phys., 2007, 46, 1579.

24 I. G. Marino, D. Bersani and P. P. Lottici, Opt. Mater., 2001, 15, 279.

25 Y. J. Wang and G. O. Carlisle, J. Mater. Sci.: Mater. Electron., 2002, 13, 173.

26 X. Zhang, S. Zhang, C. Chang, Y. Feng, Y. Li, N. Dong, K. Wang, L. Zhang, W. J. Blau and J. Wang, Nanoscale, 2015, 7, 2978.

27 J. Huang, N. Dong, S. Zhang, Z. Sun, W. Zhang and J. Wang, ACS Photonics, 2017, 4, 3063.

28 N. Dong, Y. Li, S. Zhang, X. Zhang and J. Wang, Adv. Optical Mater., 2017, 5, 1700543.
29 Y. Chen, T. Bai, N. Dong, F. Fan, S. Zhang, X. Zhuang, J. Sun, X. Zhang, J. Wang and W. J. Blau, Prog. Mater. Sci., 2016, 84, 118.

30 N. Dong, Y. Li, Y. Feng, S. Zhang, X. Zhang, C. Chang, J. Fan, L. Zhang and J. Wang, Sci. Rep., 2015, 5, 14646.

31 N. Dong, Y. Li, S. Zhang, N. McEvoy, X. Zhang, Y. Cui, L. Zhang, G. S. Duesberg and J. Wang, Opt. Lett., 2016, 41, 3936.

32 K. Wu, C. S. Guo, H. Wang, X. Y. Zhang, J. Wang and J. P. Chen, Opt. Exp., 2017, 25, 17639.

33 S. Yu, X. Wu, K. Chen, B. Chen, X. Guo, D. Dai, L. Tong, W. T. Liu and Y. Shen, Optica, 2016, 3, 541.

34 R. Yan, D. Gargas and P. Yang, Nat. Photonics, 2009, 3, 569.

35 K. L. Kompa and R. D. Levine, Proc. Natl. Acad. Sci. U. S. A., 2001, 98, 410.

36 B. Kolodziejczyk, Ch. H. Ng, X. Strakosas, G. G. Malliaras and B. Winther-Jensen, Mater. Horiz., 2018, 5, 93.

37 G. Pawlik, A. C. Mitus, J. Mysliwiec, A. Miniewicz and J. G. Grote, Chem. Phys. Lett., 2010, 484, 321.

38 A. C. Mitus, G. Pawlik, A. Kochalska, J. Mysliwiec, A. Miniewicz and F. Kajzar, Proc. SPIE, 2007, 6646, 664601.

39 H. You, H. Spaeth, V. N. L. Linhard and A. J. Steckl, Langmuir, 2009, 25, 11698.

40 Y. Kawabe, L. Wang, S. Horinouchi and N. Ogata, Adv. Mater., 2000, 12, 1281.

41 M. Dumont and A. E. Osman, Chem. Phys., 1999, 245, 437.

42 J. Mysliwiec, A. Szukalski, L. Sznitko, A. Miniewicz, K. Haupa, K. Zygadlo, K. Matczyszyn, J. Olesiak-Banska and M. Samoc, Dyes Pigm., 2014, 102, 63.

43 A. Szukalski, L. Sznitko, K. Cyprych, A. Miniewicz and J. Mysliwiec, J. Phys. Chem. C, 2014, 118, 8102.

44 J. Mysliwiec, L. Sznitko, A. Szukalski, K. Parafiniuk, S. Bartkiewicz, A. Miniewicz, B. Sahraoui, I. Rau and F. Kajzar, Opt. Mater., 2012, 34, 1725.

45 L. Sznitko, J. Mysliwiec, K. Parafiniuk, A. Szukalski, K. Palewska, S. Bartkiewicz and A. Miniewicz, Chem. Phys. Lett., 2011, 512, 247.

46 A. Miniewicz, K. Palewska, J. Lipinski, R. Kowal and B. Swedek, Mol. Cryst. Liq. Cryst., 1994, 253, 41.

47 I. Papagiannouli, A. Szukalski, K. Iliopoulos, J. Mysliwiec, S. Couris and B. Sahraoui, RSC Adv., 2015, 5, 48363.

48 A. Szukalski, B. Sahraoui, B. Kulyk, C. A. Lazar, A.-M. Manea and J. Mysliwiec, RSC Adv., 2017, 7, 9941.

49 A. Szukalski, K. Haupa, A. Miniewicz and J. Mysliwiec, J. Phys. Chem. C, 2015, 119, 10007.

50 A. Szukalski, A. Miniewicz, K. Haupa, B. Przybyl, J. Janczak, A. L. Sobolewski and J. Mysliwiec, J. Phys. Chem. C, 2016, 120, 14813.

51 M. Poprawa-Smoluch, J. Baggerman, H. Zhang, H. P. A. Maas, L. De Cola and A. M. Brouwer, J. Phys. Chem. A, 2006, 110, 11926.

52 H. Tian and J. Zhang, Photochromic Materials: Preparation, Properties and Applications, Wiley-VCH, John Wiley \& Sons, Weinheim, Germany, 2016.

53 X. Yao, T. Li, J. Wang, X. Ma and H. Tian, Adv. Opt. Mater., 2016, 4, 1322. 
54 V. Cantatore, G. Granucci and M. Persico, Phys. Chem. Chem. Phys., 2014, 16, 25081.

55 L. Brzozowski and E. H. Sargent, J. Mater. Sci.: Mater. Electron., 2001, 12, 483.

56 R. L. Sutherland, Handbook of Nonlinear Optics, Marcel Dekker Inc, New York, USA, 2nd edn, 2003.

57 R. W. Boyd, Nonlinear Optics, Academic Press, New York, 3rd edn, 2008.

58 E. Hebda, M. Jancia, F. Kajzar, J. Niziol, J. Pielichowski, I. Rau and A. Tane, Mol. Cryst. Liq. Cryst., 2012, 556, 309.

59 F. Di Benedetto, E. Mele, A. Camposeo, A. Athanassiou, R. Cingolani and D. Pisignano, Adv. Mater., 2008, 20, 314.

60 D. Bleger and S. Hecht, Angew. Chem., Int. Ed., 2015, 54, 11338.

61 J. M. Hales, J. Matichak, S. Barlow, S. Ohira, K. Yesudas, J.-L. Brédas, J. W. Perry and S. R. Marder, Science, 2010, 327, 1485.
62 Z. Li, Y. Liu, H. Kim, J. M. Hales, S.-H. Jang, J. Luo, T. Baehr-Jones, M. Hochberg, S. R. Marder, J. W. Perry and A. K.-Y. Jen, Adv. Mater., 2012, 24, OP326.

63 Z. Li, T. R. Ensley, H. Hu, Y. Zhang, S.-H. Jang, S. R. Marder, D. J. Hagan, E. W. Van Stryland and A. K.-Y. Jen, Adv. Opt. Mater., 2015, 3, 900.

64 M. Hoffmann, I. N. Papadopoulos and B. Judkewitz, Opt. Lett., 2018, 43, 22.

65 D. Li and Y. Xia, Adv. Mater., 2004, 16, 1151.

66 M. V. Kakade, S. Givens, K. Gardner, K. H. Lee, D. B. Chase and J. F. Rabolt, J. Am. Chem. Soc., 2007, 129, 2777.

67 S. Pagliara, M. S. Vitiello, A. Camposeo, A. Polini, R. Cingolani, G. Scamarcio and D. Pisignano, J. Phys. Chem. C, 2011, 115, 20399.

68 M. Richard-Lacroix and C. Pellerin, Macromolecules, 2013, 46, 9473. 